\title{
Alternative method to determine the uniaxial-strain compressibility of cohesive sandstone
}

\author{
SANDER HOL* and ARJAN VAN DER LINDEN $\dagger$
}

\begin{abstract}
Compaction and subsidence risk related to hydrocarbon production are frequently assessed using Geertsma models. The underlying theory assumes linear poroelastic reservoir rock to compress by pore pressure depletion under uniaxial-strain conditions. Adequate mechanical testing techniques have been developed to measure the uniaxial-strain compressibility, although the execution of these methods is technically difficult and requires a significant level of experience. In this paper, a simplified technique is proposed to approximate the compressibility from triaxial strain data, which is different from earlier hydrostatic stress/uniaxial-strain conversion approaches. The method is applied to Berea outcrop sandstone and Groningen reservoir material, employing pore pressure depletion protocols under triaxial stress and uniaxial-strain boundary conditions. By interpolation of triaxial stress-strain data and adopting linear poroelastic theory, the uniaxial-strain compressibility required for the application of the Geertsma model is yielded. This does not require a priori information of elastic constants. Moreover, the interpolation technique allows for an independent check of the technical performance of the radial strain control system. The method holds for rocks that are close to linear poroelastic, subjected to stress conditions below the critical state line.
\end{abstract}

KEYWORDS: compressibility; laboratory tests; rocks/rock mechanics; sands; standards \& codes of practice; stress path

\section{INTRODUCTION}

The study of rock compressibility is of major importance to applications where external stress and pore pressure are significant, and changing. Practical examples relate not only to natural tectonic mechanisms, but also to anthropogenic operations in the subsurface, notably the production of oil and gas (Donnelly, 2009; Gill \& Malamud, 2014). With the aim of quantifying risk in the subsurface, oil and gas companies conduct modelling studies. Rock compressibility is crucial for deriving the effects of reservoir compaction using, typically, the Geertsma (1973b) equation that itself is representing the deforming reservoir in the subsurface as a nucleus of strain inside an isotropic elastic half-space (cf. Eshelby, 1957). Fundamental to the treatment is the poroelastic theory by Biot (1941) that is used to describe the constitutive behaviour of the reservoir itself during depletion (pore pressure reduction during production), and the associated vertical and horizontal stress changes. For the special boundary condition of a lateral extent reservoir, the poroelastic rock is locally subjected to pore pressure depletion under zero lateral-strain boundary conditions, which results in axial compaction.

The experimental determination of compressibility has been a subject of decades-long developments in rock

Manuscript received 24 September 2018; revised manuscript accepted 13 August 2019. Published online ahead of print 16 October 2019.

Discussion on this paper closes on 1 February 2021, for further details see p. ii.

Published with permission by the ICE under the CC-BY 4.0 license. (http://creativecommons.org/licenses/by/4.0/)

* Shell Global Solutions International B.V., Hydrocarbon

Recovery Technology, Geomechanics Experimentation, Amsterdam, The Netherlands (Orcid:0000-0001-8040-618X).

$\dagger$ Shell Global Solutions International B.V., Hydrocarbon Recovery Technology, Geomechanics Experimentation, Amsterdam, The Netherlands. mechanics, starting by reducing the problem to isotropic poroelasticity (Hall, 1953; Fatt, 1958), and expanding to non-hydrostatic conditions motivated by field observations (e.g. Teufel et al., 1991). Experience with dedicated lateral strain control (Schutjens et al., 1996; Shahri \& Miska, 2014; Hol et al., 2015), in order to fulfil the boundary condition requirement, has resulted in the recent publication of a suggested method for uniaxial-strain compressibility testing by the International Society for Rock Mechanics (ISRM) (Dudley et al., 2016). Despite their usefulness and necessity, the protocols are challenging in terms of calibration and execution and require a high level of experience of the operating staff. As a consequence, tests are usually expensive, and results not always reliable.

In this paper, the authors describe how, for cohesive poroelastic rock subjected to stress below the critical state line, the axial compressibility can be determined by the execution of two (technically less challenging) triaxial pore pressure depletion tests close to the expected in situ stress change, from which the uniaxial-strain compressibility can be inferred. This technique is successfully applied using two different rocks, notably a Berea outcrop sandstone and a reservoir sandstone from the Groningen Field, The Netherlands.

\section{MATERIAL AND METHODS}

A linear poroelastic material subjected to hydrostatic stress and pressure has four intrinsic compressibilities (Zimmerman, 1991) defined in terms of the change in bulk volume $V_{\mathrm{b}}$ and pore volume $V_{\mathrm{p}}$ in response to a change in either confining pressure $P_{\mathrm{c}}$ or pore pressure $P$. When dealing with problems in the brittle crust - for example, reservoir compaction associated with fluid extraction - rock deformation in the field must be extended to non-hydrostatic poroelasticity, which introduces the problem of threedimensionality in stress-strain response (e.g. Biot, 1941; Bouteca, 1992; Wang, 2000). Burbey (1999) compared in detail the role of uniaxial-strain boundary conditions 
(Jacob, 1940) with the triaxial stress boundary condition (Biot, 1941) for aquifer depletion. Here, the focus is placed specifically on the experimental characterisation of axial compressibility required for compaction and subsidence modelling. The case of uniaxial-strain compression of oil and gas reservoirs is treated by Geertsma (1973a). Specific to this problem, the current authors have first derived in the Appendix a set of practical equations expressing the axial compressibility in more general terms for triaxial stress boundary condition

$$
\begin{gathered}
\Delta e_{1}=-\frac{2 v}{E}\left(\sigma_{3,1}-\sigma_{3, \mathrm{ref}}\right)+\frac{\alpha(1-2 v)}{E}\left(P_{1}-P_{\mathrm{ref}}\right) \rightarrow \\
C_{1-\text { triaxial }}=\frac{\Delta e_{1}}{\Delta P}=-\frac{2 v}{E} \frac{\Delta \sigma_{3}}{\Delta P}+\frac{\alpha(1-2 v)}{E}
\end{gathered}
$$

(this equation is derived and presented as equation (12) in the Appendix) and uniaxial-strain boundary conditions

$$
\begin{aligned}
& \Delta e_{1}=-\frac{2 v}{E} \Delta \sigma_{3}+\frac{\alpha(1-2 v)}{E} \Delta P \rightarrow \\
& C_{1-\text { uniaxial }}=C_{\mathrm{m}}=\frac{\Delta e_{1}}{\Delta P}=-\frac{2 v \gamma_{\mathrm{h}}}{E}+\frac{\alpha(1-2 v)}{E}
\end{aligned}
$$

(this equation is derived and presented as equation (14) in the Appendix). Using expressions (1) and (2), several cohesive sandstone samples are subjected to a pore pressure depletion protocol under these two boundary conditions (Fig. 1). First, the authors interpolate from the radial strain response the $\gamma_{\mathrm{h}}$ required to maintain uniaxial-strain boundary conditions. A control test is then performed, subjecting a sample to true uniaxial-strain pore pressure depletion, in order to check this assessment of the $\gamma_{\mathrm{h}}$, and predicted $C_{\mathrm{m}}$. The tests are performed using samples taken from Berea outcrop material (indicator 'BER-18'), and from research well Zeerijp-3A located in the Groningen Field, The Netherlands (indicator 'ZRP-3A'). Stress conditions applied during the mechanical experiments with the ZRP-3A samples were consistent with stresses expected in the reservoir, which were also taken as a basis for the conditions applied during the tests using BER-18 material. The experiments using ZRP-3A material were reported previously (Hol et al., 2018). All experiments are carried out at room temperature.
Material properties and pore fluid composition

Plug samples were drilled vertically from either 4 inch $(10 \cdot 16 \mathrm{~cm})$ full core material (ZRP-3A), or from a block of outcrop material (BER-18). All samples were prepared with dimensions 1 inch $(2.54 \mathrm{~cm})$ in diameter and 2 inches $(5.08 \mathrm{~cm})$ long, which is required to prevent endcap friction effects in the present tests (Dudley et al., 2016). The BER-18 samples were drilled using tap water as the cooling and lubrication fluid, and then dried in an oven at $95^{\circ} \mathrm{C}$ and $200 \mathrm{mbar}$ vacuum for $24 \mathrm{~h}$. To avoid possible clay swelling, the ZRP-3A samples were drilled using the synthetic reservoir brine as cooling and lubrication fluid. Porosity was determined by means of the buoyancy method using mercury and chloroform, where the bulk volume is checked against caliper measurements (see Table 2 later). Note that the porosity of all samples is in the range $19-21 \%$, and the permeability is roughly between $110 \mathrm{mD}$ and $210 \mathrm{mD}$, with an increase in permeability with increasing porosity. Since both porosity and permeability are fundamental properties in the mechanical behaviour of rocks under conditions of pore pressure depletion, these measurements give confidence in that the selected samples are indeed suitable for pore pressure depletion testing under the (changing) boundary conditions chosen, and that the relative difference in properties is small enough to allow for a comparison of mechanical data between samples. After drying and basic core analysis, the samples were vacuum saturated with synthetic brine with the same composition as the Groningen reservoir formation brine (Table 1).

\section{Experimental method}

The BER-18 and ZRP-3A sandstone samples are subjected to pore pressure depletion under triaxial stress and uniaxial-strain boundary conditions. The experimental conditions controlled and measured are the axial stress $S_{\text {ax }}$, which equals $\sigma_{1}$, the radial stress $S_{\text {rad }}$, which equals $\sigma_{2}$ and $\sigma_{3}$, and pore pressure $P$. The stress path chosen follows a pore pressure depletion protocol, whereby the experimental conditions of $S_{\mathrm{ax}}, S_{\mathrm{rad}}, P$ against time are based on the stress and pore pressure conditions listed in Table 2. These are all applied at a rate of $2.5 \mathrm{MPa} / \mathrm{h}$, using a conventional triaxial systems (refer to Hol et al. (2018)). The hydraulic pump systems used to apply $S_{\mathrm{ax}}$ and $S_{\text {rad }}$ can be measured

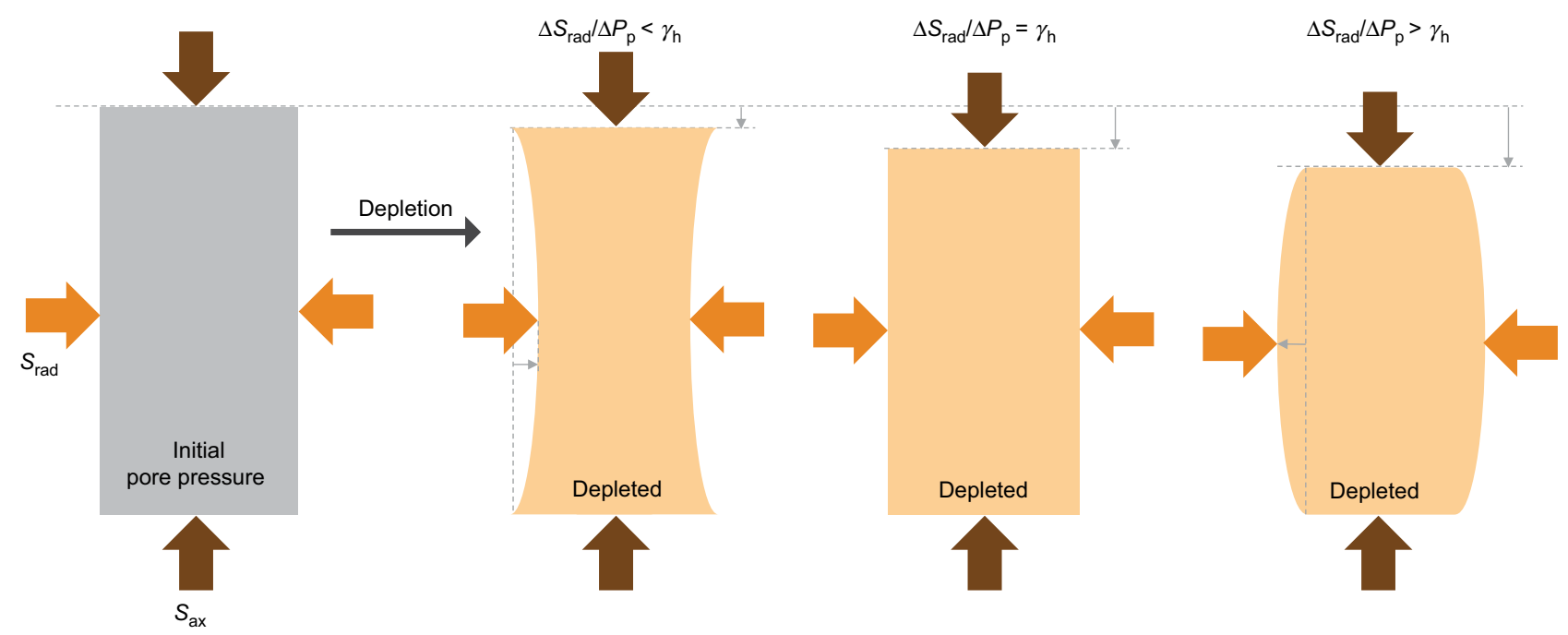

Fig. 1. Schematic drawing of the concept of testing. A sample is initially exposed to triaxial stress and pore pressure, and then subjected to pore pressure change to arrive at one of the three final states at the right. Axial and radial strains are measured continuously during testing to allow for the application of equations (1), (2) and (13) 
Table 1. Composition of the synthetic brine used in this study, based on formation brine composition for the Groningen Field

\begin{tabular}{l|c}
\hline Component & Concentration: $\mathrm{g} / \mathrm{l}$ \\
\hline Sodium chloride $(\mathrm{NaCl})$ & $197 \cdot 71$ \\
Potassium chloride $(\mathrm{KCl})$ & $4 \cdot 44$ \\
Calcium chloride dihydrate $\left(\mathrm{CaCl}_{2} \cdot 2 \mathrm{H}_{2} \mathrm{O}\right)$ & $147 \cdot 84$ \\
Magnesium chloride hexahydrate & $25 \cdot 10$ \\
$\quad\left(\mathrm{MgCl}_{2} \cdot 6 \mathrm{H}_{2} \mathrm{O}\right)$ & $5 \cdot 20$ \\
Strontium chloride hexahydrate & \\
$\quad\left(\mathrm{SrCl}_{2} \cdot 6 \mathrm{H}_{2} \mathrm{O}\right)$ & \\
\hline
\end{tabular}

and controlled to within $0 \cdot 1 \mathrm{MPa}$, up to $100 \mathrm{MPa}$ pressure. It is important to note that the piston area equals the sample area so that the hydraulic pump pressure is equal to the applied $S_{\mathrm{ax}}$. Pore fluid pressure $P$ can be applied to the sample, at the top and bottom piston, and can be either brine or oil. Axial displacement is measured with an accuracy of $\pm 0 \cdot 11 \mu \mathrm{m}$ using a linear variable differential transformer (LVDT) and is used to compute the axial strain $e_{\mathrm{ax}}$. Radial strain $e_{\text {rad }}$ is measured with an accuracy of $\pm 0 \cdot 1 \mu \mathrm{m}$ using a cantilever bridge wired as a full Wheatstone bridge, and can also be used as input to the confining pressure control. This system is positioned onto two pins that are in direct contact with the sample, 1 inch $(2.54 \mathrm{~cm})$ from the sample top and bottom - that is exactly in the middle, where no strain effects due to endcap friction are expected. All experiments are executed in a fully automated manner, using computer-scripted protocols. A periodic calibration of all sensors is performed to ensure accurate measurement and control of all signals, including a necessary correction for apparatus distortion effects, in particular because these are part of the uniaxial-strain control feedback loop.

When setting up an experiment, a sample is placed in a flexible elastomer sleeve and mounted in the compaction cell, with porous plates at the top and bottom of the sample to facilitate pore fluid saturation and communication. Then, the sample is brought to a slightly elevated stress of $S_{\text {ax }}=1.5 \mathrm{MPa}$, and $S_{\text {rad }}=1 \mathrm{MPa}$, to allow for settlement of the sleeve and sensors. Brine is flushed through the pore fluid system at $P=0.5 \mathrm{MPa}$ to ensure saturation of the sample. After saturation, the samples are subject to a specific pore pressure depletion protocol, either employing triaxial stress control or uniaxial-strain control.

Test protocols for BER-18 samples. First, $S_{\mathrm{ax}}, S_{\mathrm{rad}}$ and $P$ are increased simultaneously by $10 \mathrm{MPa}$ - that is increased to achieve isostatic external stress and pore pressure. Then, $S_{\text {ax }}$ and $S_{\text {rad }}$ are raised by another $10 \mathrm{MPa}$, followed again by an increase of $S_{\mathrm{ax}}$ by $10 \mathrm{MPa}$ while keeping $S_{\mathrm{rad}}$ and $P$ constant. Then, all stresses are increased to those listed in Table 2. After a settling period of approximately $12 \mathrm{~h}, S_{\mathrm{rad}}$ and $P$ are decreased with a fixed relative $\Delta S_{\text {rad }} / \Delta P$ ratio while keeping $S_{\mathrm{ax}}$ constant. The ratio chosen aims at being higher and lower than $\gamma_{\mathrm{h}}$, hence allowing for its approximation by interpolation. After consolidation at $P_{\text {dep }}$ (Table 2), the initial $S_{\mathrm{ax}}, S_{\mathrm{rad}}$ and $P_{\text {ini }}$ are restored by re-pressurisation, followed by a second pore pressure depletion step. Both re-pressurisation and the second depletion stage are performed with the same fixed relative $\Delta S_{\text {rad }} / \Delta P$ ratio while keeping $S_{\mathrm{ax}}$ constant. In addition, two experiments were performed with the depletion and re-pressurisation cycles under true uniaxial-strain boundary conditions. In this case, $S_{\text {rad }}$ is controlled to maintain zero-lateral strain conditions during depletion and re-pressurisation. An overview of the experiments, and the applied $\Delta S_{\mathrm{rad}} / \Delta P$ ratios, is presented in Table 3. An example of a triaxial pore pressure depletion protocol is presented in Fig. 2, in this case for an experiment in which a $\Delta S_{\text {rad }} / \Delta P$ ratio is employed of $0 \cdot 4$.

Test protocols for ZRP-3A samples. In a manner similar to the BER-18 samples, the ZRP-3A samples were first brought to the pre-depletion stress and pore pressure condition that approaches the virgin in situ condition of stress and pressure in the Groningen Field - that is $S_{\mathrm{ax}}, S_{\mathrm{rad}}$ and $P$ are raised to the pre-depletion conditions listed in Table 2. After $48 \mathrm{~h}$ of mechanical settling, $P$ is decreased in cycles under actively controlled triaxial stress, or uniaxial-strain boundary conditions, until maximum depletion at $3 \mathrm{MPa}$ pore pressure was achieved (Fig. 3). Each depletion step of $10 \mathrm{MPa}$ is followed by three re-pressurisation and depletion cycles of $5 \mathrm{MPa}$ to allow for a comparison of the depletion and re-pressurisation responses. An overview of the experiments is listed in Table 3, accompanied by the applied $\Delta S_{\text {rad }} / \Delta P$ ratios. An example of a uniaxial-strain pore pressure depletion protocol is presented in Fig. 3, including the three re-pressurisation-depletion cycles employed in this protocol. Although the protocol employed in the case of the BER-18 samples consists of one-step depletion and re-pressurisation cycles, the testing principle applied in the case of the ZRP-3A samples is identical in that it systematically compares loading and unloading behaviour. The ZRP-3A protocol was chosen to best represent the true in situ depletion path while allowing for an assessment of the elastic response by means of unloading. It merely serves the

Table 2. Overview of stress conditions, porosity and permeability, for all BER-18 and ZRP-3A samples

\begin{tabular}{l|c|l|l|l|c|c|c|c|c}
\hline Sample ID & $\begin{array}{c}S_{\text {ax }}: \\
\text { MPa }\end{array}$ & $\begin{array}{c}S_{\text {rad: }}: \\
\text { MPa }\end{array}$ & $\begin{array}{c}P_{\text {ini }}: \\
\text { MPa }\end{array}$ & $\begin{array}{c}P_{\text {dep }}: \\
\text { MPa }\end{array}$ & $\begin{array}{c}\text { Porosity: } \\
\%\end{array}$ & $\begin{array}{c}\text { Density solid phase } \\
\text { (grain density): g/ml }\end{array}$ & $\begin{array}{c}\text { Density } \\
\text { bulk: g/ml }\end{array}$ & $\begin{array}{c}\text { Permeability } \\
\text { to N } \mathrm{N}_{2}: \mathrm{mD}\end{array}$ & $\begin{array}{c}\text { Permeability } \\
\text { Klinkenberg } \\
\text { corrected: } \mathrm{mD}\end{array}$ \\
\hline BER-18_47EV & 60 & 50 & 40 & 10 & $20 \cdot 1$ & $2 \cdot 656$ & $2 \cdot 123$ & 142 & 138 \\
BER-18_48EV & 60 & 50 & 40 & 10 & $19 \cdot 6$ & $2 \cdot 656$ & $2 \cdot 137$ & 115 & 112 \\
BER-18_50EV & 60 & 50 & 40 & 10 & $20 \cdot 6$ & $2 \cdot 656$ & $2 \cdot 110$ & 175 & 171 \\
BER-18_51EV & 60 & 50 & 40 & 10 & $20 \cdot 7$ & $2 \cdot 656$ & $2 \cdot 106$ & 176 & 172 \\
BER-18_52EV & 60 & 50 & 40 & 10 & $20 \cdot 4$ & $2 \cdot 656$ & $2 \cdot 115$ & 179 & 175 \\
BER-18_54EV & 60 & 50 & 40 & 10 & $20 \cdot 8$ & $2 \cdot 656$ & $2 \cdot 103$ & 182 & 178 \\
BER-18_55EV & 60 & 50 & 40 & 10 & $19 \cdot 6$ & $2 \cdot 657$ & $2 \cdot 136$ & 122 & 119 \\
BER-18_56EV & 60 & 50 & 40 & 10 & $21 \cdot 0$ & $2 \cdot 657$ & $2 \cdot 100$ & 206 & 202 \\
BER-18_57EV & 60 & 50 & 40 & 10 & $20 \cdot 9$ & $2 \cdot 657$ & $2 \cdot 100$ & 215 & 211 \\
ZRP-3A_10EV & 69 & 57 & $35 \cdot 5$ & 3 & $20 \cdot 8$ & $2 \cdot 632$ & $2 \cdot 084$ & 129 & 117 \\
\hline
\end{tabular}

Stresses and pressure listed are the vertical stress $S_{\text {ax }}$, initial radial stress $S_{\text {rad, }}$, initial pore pressure $P_{\text {ini }}$ and pore pressure in the depleted state $P_{\text {dep }}$. The permeability to nitrogen $\left(\mathrm{N}_{2}\right)$ is corrected for gas slippage and turbulence effects 
Table 3. Overview of tested BER-18 and ZRP3A samples, with corresponding control mode, and applied $\Delta S_{\text {rad }} / \Delta P$ ratio or measured $\gamma_{\mathrm{h}}$

\begin{tabular}{|c|c|c|c|c|}
\hline Sample ID & Control mode & $\begin{array}{l}\Delta S_{\mathrm{rad}} / \Delta P \text { ratio } \\
\text { first depletion stage }\end{array}$ & $\begin{array}{l}\Delta S_{\mathrm{rad}} / \Delta P \text { ratio } \\
\text { first re-pressurisation stage }\end{array}$ & $\begin{array}{l}\Delta S_{\text {rad }} / \Delta P \text { ratio } \\
\text { second depletion stage }\end{array}$ \\
\hline $\begin{array}{l}\text { BER-18_57EV } \\
\text { BER-18_47EV } \\
\text { BER-18_50EV } \\
\text { BER-18_48EV } \\
\text { BER-18_54EV } \\
\text { BER-18_56EV } \\
\text { BER-18_52EV } \\
\text { BER-18_51EV } \\
\text { BER-18_55EV }\end{array}$ & $\begin{array}{l}\text { Triaxial stress } \\
\text { Triaxial stress } \\
\text { Triaxial stress } \\
\text { Triaxial stress } \\
\text { Triaxial stress } \\
\text { Triaxial stress } \\
\text { Triaxial stress } \\
\text { Uniaxial-strain } \\
\text { Uniaxial-strain }\end{array}$ & $\begin{array}{l}0 \cdot 0 \\
0 \cdot 4 \\
0 \cdot 4 \\
0 \cdot 74 \\
0 \cdot 8 \\
0 \cdot 8 \\
1 \cdot 0 \\
\text { Measured } \gamma_{\mathrm{h}} \\
\text { Measured } \gamma_{\mathrm{h}}\end{array}$ & $\begin{array}{l}0 \cdot 0 \\
0 \cdot 4 \\
0 \cdot 4 \\
0 \cdot 65 \\
0 \cdot 8 \\
0 \cdot 8 \\
1 \cdot 0 \\
\text { Measured } \gamma_{\mathrm{h}} \\
\text { Measured } \gamma_{\mathrm{h}}\end{array}$ & $\begin{array}{l}0 \cdot 0 \\
0 \cdot 4 \\
0 \cdot 4 \\
0 \cdot 65 \\
0 \cdot 8 \\
0 \cdot 8 \\
1 \cdot 0 \\
\text { Measured } \gamma_{\mathrm{h}} \\
\text { Measured } \gamma_{\mathrm{h}}\end{array}$ \\
\hline Sample ID & Control mode & $\begin{array}{l}\Delta S_{\text {rad }} / \Delta P \text { ratio primary } \\
\text { depletion step }\end{array}$ & $\begin{array}{l}\Delta S_{\text {rad }} / \Delta P \text { ratio } \\
\text { re-pressurisation-depletion } \\
\text { cycles }\end{array}$ & \\
\hline $\begin{array}{l}\text { ZRP-3A_10AV } \\
\text { ZRP-3A_10BV } \\
\text { ZRP-3A_10CV } \\
\text { ZRP-3A_10DV }\end{array}$ & $\begin{array}{l}\text { Uniaxial-strain } \\
\text { Uniaxial-strain } \\
\text { Triaxial stress } \\
\text { Triaxial stress }\end{array}$ & $\begin{array}{l}\text { Measured } \gamma_{\mathrm{h}} \\
\text { Measured } \gamma_{\mathrm{h}} \\
0 \cdot 5 \\
0.9\end{array}$ & $\begin{array}{l}\text { Measured } \gamma_{\mathrm{h}} \\
\text { Measured } \gamma_{\mathrm{h}} \\
0 \cdot 5 \\
0 \cdot 9\end{array}$ & \\
\hline
\end{tabular}

Samples are ordered by control mode, $\Delta S_{\text {rad }} / \Delta P$ value. Note that the ratios for BER-18_48EV were not identical for control reasons

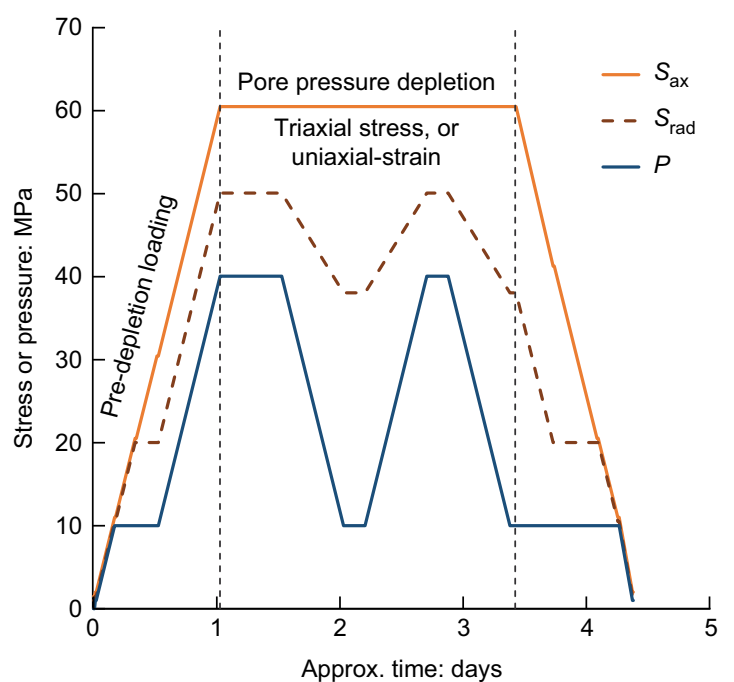

Fig. 2. Example of the triaxial pore pressure depletion protocol, in this case with a $\Delta S_{\mathrm{rad}} / \Delta P$ ratio of $0 \cdot 4$, applied to samples BER-18_47EV and BER-18_50 (refer to Table 3)

purpose of a control test to test the applicability of the triaxial stress test method to determine $C_{1 \text {-uniaxial }}$ or $C_{\mathrm{m}}$.

\section{Data processing}

All measurements of $S_{\mathrm{ax}}, S_{\mathrm{rad}}$ and $P$, as well as axial and radial displacements $\Delta l_{\mathrm{ax}}$ and $\Delta l_{\mathrm{rad}}$, were logged at a resolution of 12-bit using a computer. Using the initial sample length $l_{0}$, the axial engineering strain $e_{\mathrm{ax}}$ and $e_{\text {rad }}$ are computed to compare the $e_{\mathrm{ax}}, e_{\mathrm{rad}}, S_{\mathrm{ax}}$ and $S_{\mathrm{rad}}$ responses as a function of changing pore pressure $\Delta P$ during depletion. The purpose is to compare these properties, and check whether the responses satisfy the poroelastic stress-strain relations for triaxial stress and uniaxial-strain boundary conditions. In the case of the BER-18 samples (Fig. 2), the authors choose to parameterise the first depletion step (PPD\#1), the first re-pressurisation step (PPR\#1) and the second depletion step (PPD\#2). The experimental data measured for the ZRP-3A samples (Fig. 3) are parameterised

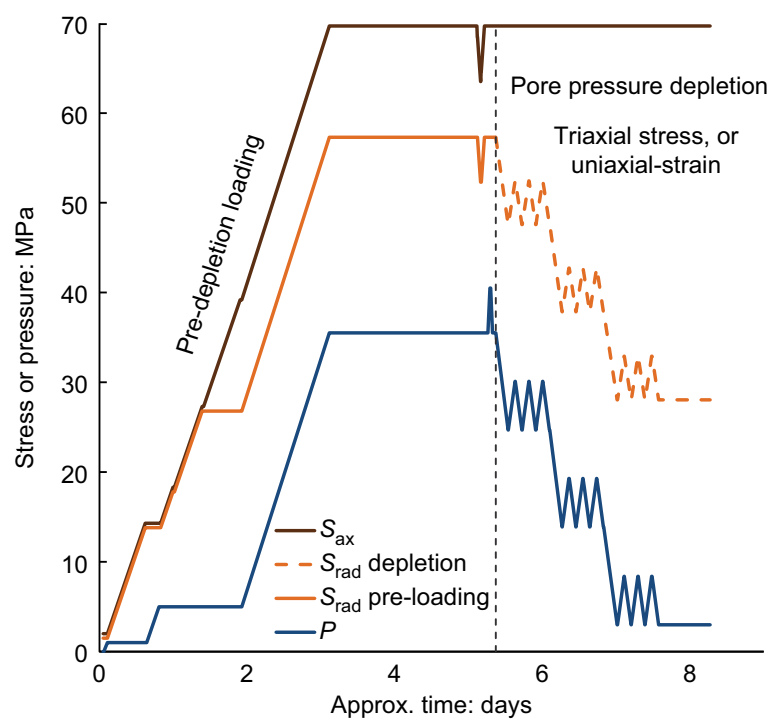

Fig. 3. Example of the triaxial pore pressure depletion protocol, in this case with a $\Delta S_{\mathrm{rad}} / \Delta P$ ratio of 0.9 , applied to sample ZRP-3A_10DV (refer to Table 3)

over the total depletion step from $35 \mathrm{MPa}$ to $3 \mathrm{MPa}$ pore pressure (PPDtot), as well as over each individual step - that is 35-25 $\mathrm{MPa}$ (PPD35-25), 25-15 $\mathrm{MPa}$ (PPD25-15) and 15-3 MPa (PPD15-3). Re-pressurisation steps are also parameterised - that is the steps 25-30 MPa (PPR25-30), 15-20 MPa (PPR 15-20) and 3-7 MPa (PPR3-7) - but solely used to compute the fractional contribution of elastic strain $e_{\mathrm{el}}$.

\section{RESULTS}

In total, 13 experiments were performed using the BER-18 and ZRP-3A samples, employing pore pressure depletion protocols either under conditions of triaxial stress (fixed $\Delta S_{\mathrm{rad}} / \Delta P$ ratio), or uniaxial-strain (measuring $\gamma_{\mathrm{h}}$ in response). The parameterised $e_{\mathrm{ax}}, e_{\mathrm{rad}}$ and $S_{\mathrm{rad}}$ data are provided in the online supplementary material, and these data are plotted systematically later in Figs 5-7, to identify 
Table 4. Regression statistics for relations between strain components $e_{\mathrm{ax}}$ and $e_{\text {rad }}$ measured for BER-18 samples during depletion step PPD\#1, re-pressurisation step PPR\#1 and depletion step PPD\#2, and for ZRP-3A samples measured during the cyclic depletion steps

\begin{tabular}{|c|c|c|c|c|c|}
\hline BER-18 test cycles & Intercept (strain) & Slope (strain per $\Delta S_{\mathrm{rad}} / \Delta P$ ratio) & $p$-value & FStat & $R^{2}$ \\
\hline $\begin{array}{l}\text { e-rad - (PPD\#1) } \\
\text { e-rad - (PPR\#1) } \\
\text { e-rad - (PPD\#2) } \\
\text { e-ax - (PPD\#1) } \\
\text { e-ax - (PPR\#1) } \\
\text { e-ax - (PPD\#2) }\end{array}$ & $\begin{array}{r}1.28 \times 10^{-3} \\
-0.97 \times 10^{-3} \\
0.99 \times 10^{-3} \\
1.06 \times 10^{-3} \\
-0.52 \times 10^{-3} \\
0.56 \times 10^{-3}\end{array}$ & $\begin{array}{r}-1.63 \times 10^{-3} \\
1.36 \times 10^{-3} \\
-1.37 \times 10^{-3} \\
1.56 \times 10^{-3} \\
-1.79 \times 10^{-3} \\
1.76 \times 10^{-3}\end{array}$ & $\begin{array}{l}3 \cdot 32 \times 10^{-5} \\
3 \cdot 01 \times 10^{-4} \\
2 \cdot 41 \times 10^{-4} \\
1 \cdot 94 \times 10^{-6} \\
2 \cdot 14 \times 10^{-5} \\
1 \cdot 57 \times 10^{-5}\end{array}$ & $\begin{array}{r}69 \cdot 02 \\
55 \cdot 54 \\
60 \cdot 17 \\
115 \cdot 72 \\
99 \cdot 94 \\
109 \cdot 88\end{array}$ & $\begin{array}{l}0 \cdot 90 \\
0 \cdot 90 \\
0 \cdot 91 \\
0 \cdot 93 \\
0 \cdot 93 \\
0 \cdot 94\end{array}$ \\
\hline ZRP-3A test cycles & Intercept (strain) & Slope (strain per $\Delta S_{\mathrm{rad}} / \Delta P$ ratio) & $p$-value & FStat & $R^{2}$ \\
\hline $\begin{array}{l}\text { e-rad - (PPDtot) } \\
\text { e-rad - (PPD35-25) } \\
\text { e-rad - (PPD25-15) } \\
\text { e-rad - (PPD15-3) } \\
\text { e-ax - (PPDtot) } \\
\text { e-ax - (PPD35-25) } \\
\text { e-ax - (PPD25-15) } \\
\text { e-ax - (PPD15-3) }\end{array}$ & $\begin{array}{l}3 \cdot 23 \times 10^{-3} \\
0 \cdot 77 \times 10^{-3} \\
1 \cdot 96 \times 10^{-3} \\
3 \cdot 03 \times 10^{-3} \\
1 \cdot 34 \times 10^{-3} \\
0 \cdot 23 \times 10^{-3} \\
0 \cdot 18 \times 10^{-3} \\
0 \cdot 19 \times 10^{-3}\end{array}$ & $\begin{array}{r}-3 \cdot 94 \times 10^{-3} \\
-1 \cdot 03 \times 10^{-3} \\
-2 \cdot 55 \times 10^{-3} \\
-3 \cdot 90 \times 10^{-3} \\
2 \cdot 47 \times 10^{-3} \\
1 \cdot 00 \times 10^{-3} \\
1 \cdot 14 \times 10^{-3} \\
1 \cdot 04 \times 10^{-3}\end{array}$ & $\begin{array}{l}1 \cdot 09 \times 10^{-3} \\
2 \cdot 08 \times 10^{-5} \\
3 \cdot 21 \times 10^{-3} \\
1 \cdot 38 \times 10^{-3} \\
2 \cdot 05 \times 10^{-1} \\
1 \cdot 85 \times 10^{-1} \\
1 \cdot 23 \times 10^{-1} \\
1 \cdot 47 \times 10^{-1}\end{array}$ & $\begin{array}{r}916 \cdot 48 \\
012 \cdot 08 \\
310 \cdot 15 \\
721 \cdot 96 \\
3 \cdot 44 \\
3 \cdot 97 \\
6 \cdot 63 \\
5 \cdot 33\end{array}$ & $\begin{array}{l}1 \cdot 00 \\
1 \cdot 00 \\
0 \cdot 99 \\
1 \cdot 00 \\
0 \cdot 63 \\
0 \cdot 66 \\
0 \cdot 77 \\
0 \cdot 73\end{array}$ \\
\hline
\end{tabular}

Also listed are the slopes and strain intercepts. Note the low $p$-values in general (smaller than 0.05 for BER-18 strains and ZRP-3A radial strains), and the high correlation coefficient $R^{2}$ (better than 0.9 for all correlations). Measurements of $e_{\mathrm{ax}}$ for the ZRP-3A data correlate less well, which results in uncertainty in the prediction of $e_{\mathrm{ax}}$, and hence $C_{1 \text {-uniaxial }}$, or $C_{\mathrm{m}}$

whether the linear poroelastic relationships in equations (1) and (2) exist for the measured stress and strain responses. Regression statistics are provided in Table 4.

\section{Response to pre-depletion loading}

Loading to initial stress and pore pressure conditions (Table 2) was performed for all samples in an identical manner to pre-condition the material. From the measured $e_{\mathrm{ax}}$, in response to the increase of $S_{\mathrm{ax}}$ under conditions of constant $S_{\mathrm{rad}}$ and $P$ (refer to Fig. 4 for selected BER-18 samples), the Young's modulus $E$ was determined. This was practically achieved by taking the tangent of the $S_{\mathrm{ax}}-e_{\mathrm{ax}}$ curve. The procedure yielded for the BER-18 samples values for $E$ in the range from $7 \mathrm{GPa}$ to $10 \mathrm{GPa}$, with a mean $E$ of $8 \cdot 3 \mathrm{GPa} \pm 0 \cdot 8 \mathrm{GPa}$, and for the ZRP-3A samples values for $E$ in the range from $6 \mathrm{GPa}$ to $8 \mathrm{GPa}$, with a mean $E$ of $6.7 \mathrm{GPa} \pm 0.8 \mathrm{GPa}$. Although perhaps affected by the closure of cracks in the pre-depletion part of the loading protocol, the data show that $E$ is closely similar for all samples tested within a particular series, and consistent with values reported in the literature (e.g. Hart \& Wang, 1995; Delle Piane \& Sarout, 2016).

\section{Stress-strain response to depletion of BER-18 samples}

Depletion and re-pressurisation of BER-18 samples was performed in uninterrupted steps, from the adopted initial pore pressure $P_{\text {ini }}$ to the full depletion pressure $P_{\text {dep }}$ (refer to Fig. 4, and the online supplementary material). Axial and radial strains responded to the pore pressure change in a closely linear manner (Fig. 4), and the total radial strain $e_{\text {rad }}$ (Fig. 5) and axial strain $e_{\mathrm{ax}}$ (Fig. 6) for each step are strongly related to the radial stress change $\Delta S_{\text {rad }}$ imposed (triaxial stress), or measured (uniaxial-strain). The subsequent pore pressure re-pressurisation and second depletion steps, performed under the same controlled radial stress change, resulted in nearly complete recoverability of the axial strain for most samples (Fig. 4). Strains are generally decreasing in magnitude with depletion step (compare PPR\#1 and PPD\#2, with PPD\#1 in Figs 5 and 6), which implies that the samples are stiffening during the PPD\#1 step. Exceptions to general behaviour were the experiments carried out with an imposed $\Delta S_{\text {rad }} / \Delta P$ ratio of zero (BER-18_57EV and BER-18_58EV). In these cases, the magnitude of radial strain was equal to (BER-18_58EV) or twice (BER-18_57EV) the axial strain, which demonstrates that the unrealistic boundary condition had resulted in radial overstressing of the sample. Comparing the loading (PPD\#1) and unloading cycles (PPR\#1) of these tests, shows that only a factor $0 \cdot 3$ to $0 \cdot 5$ of the strains was recoverable. The opposite end-member case, where the $\Delta S_{\mathrm{rad}} / \Delta P$ ratio is unity (sample Ber18_52EV), resulted in relatively high positive axial strain $\left(e_{\mathrm{ax}}=0.0023\right.$, Fig. 4$)$, accompanied by negative radial strain $\left(e_{\mathrm{rad}}=-0.0005\right.$, Fig. 4$)$, implying that the sample deformed by axial compression and radial expansion. This is, in terms of effective stress change, similar to the mode of deformation in a conventional triaxial compressive strength test. All remaining experiments were carried out by choosing a fixed $\Delta S_{\mathrm{rad}} / \Delta P$ ratio between zero and unity (Table 3 ), which resulted in positive (compressive) to negative (expansional) radial strain (Fig. 5), and positive yet decreasing axial strain (Fig. 6), as a function of the imposed $\Delta S_{\mathrm{rad}} / \Delta P$ ratio.

Forcing now a linear regression onto the data presented in Figs 5 and 6, in this case including samples BER-18_58EV and BER-18_57EV, shows that a linear relation must exist between the axial and radial stress-strain components as a function of changing pore pressure, independent of any intrinsic change (non-linearity) in elastic constants during loading. The radial strain response plotted in Fig. 5 displays a positive and negative response, divided by the intercept with the $x$-axis. This intercept represents the $\Delta S_{\text {rad }} / \Delta P$ ratio where no radial strain takes place during the employed pore pressure depletion steps - that is the point represents the radial stress change required to achieve uniaxial-strain conditions. Indeed, the intercepts of PPD\#1 (0.77), PPR\#1 $(0 \cdot 70)$ and PPD\#2 (0.72) are consistent with the values for $\gamma_{\mathrm{h}}$ measured at true uniaxial-strain boundary conditions (in the range $0 \cdot 70-0 \cdot 80$ ). Moreover, the axial strain plotted against the imposed or measured $\Delta S_{\text {rad }} / \Delta P$ ratio, shown in Fig. 6, shows a clear linear relation, which is either positive in the case of depletion, or negative in the case of re-pressurisation (Fig. 6). The linear relation clearly shows that the uniaxialstrain experiments are part of the same trend. All $p$-values for 


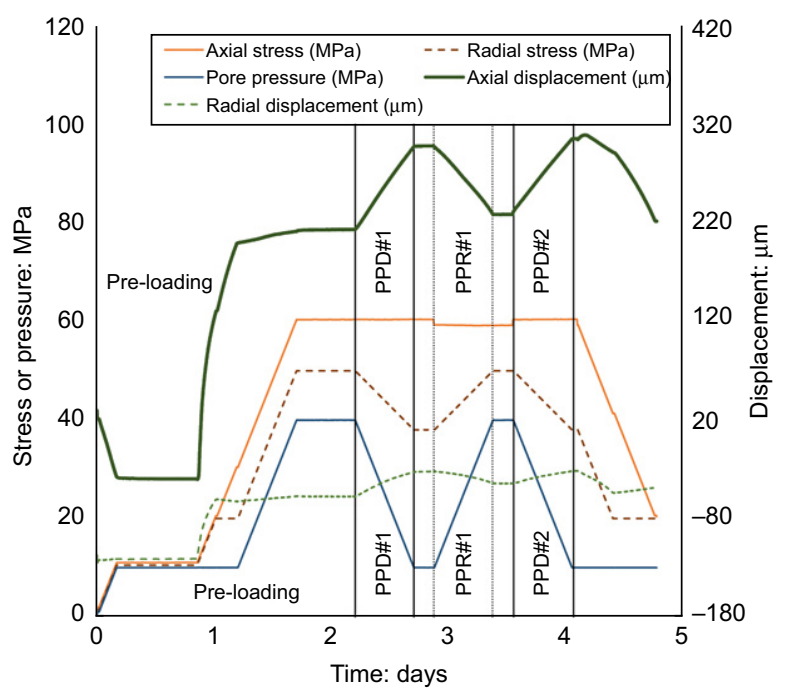

(a)

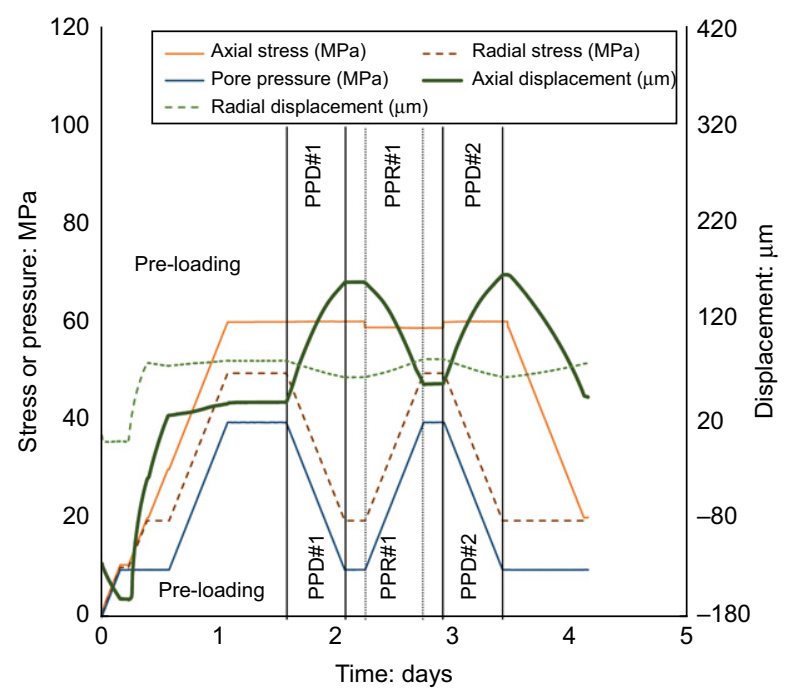

(c)

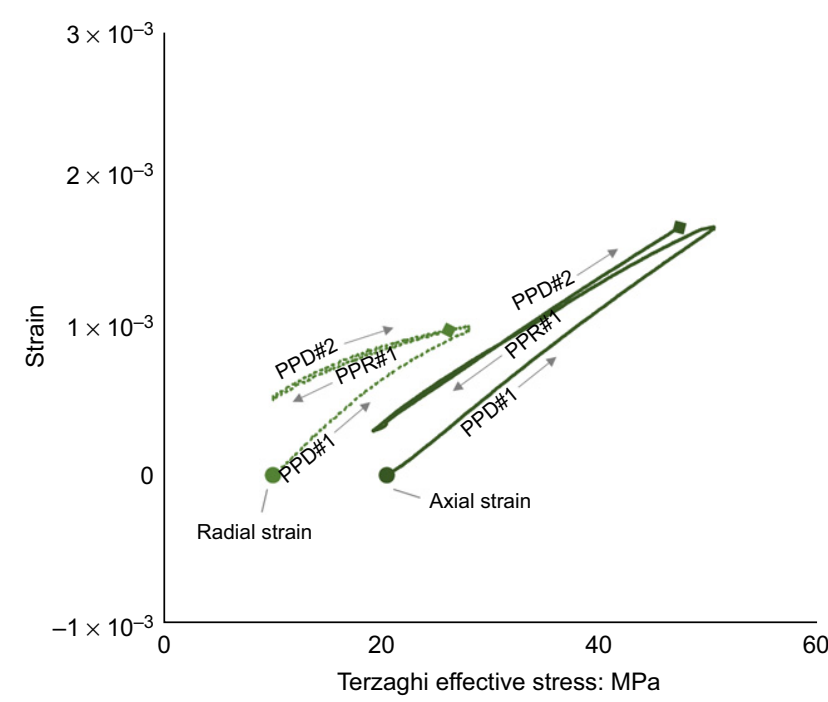

(b)

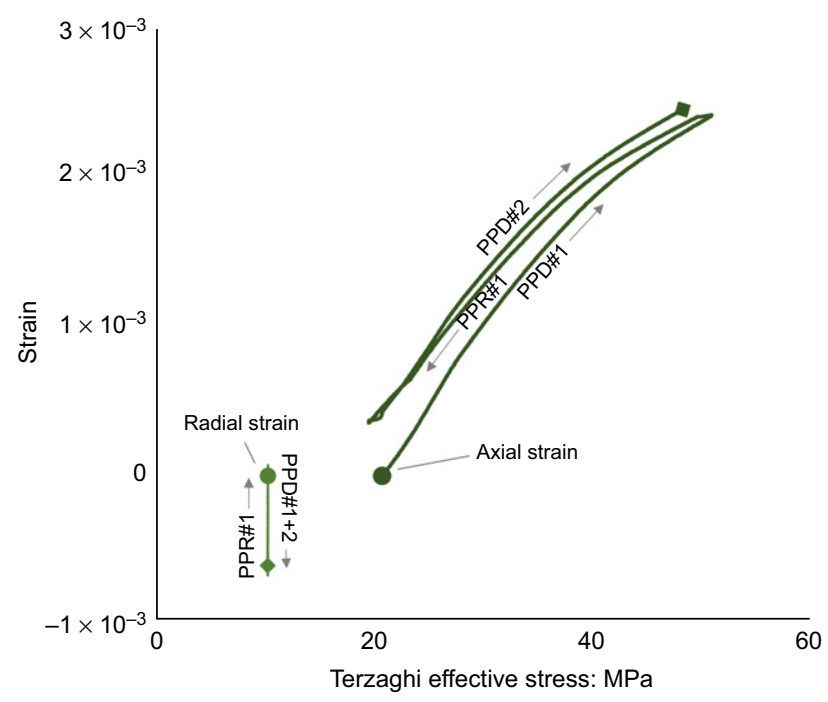

(d)

Fig. 4. Displacement/strain response and measured stress and pore pressure data against time (in days) for sample BER18_47EV and sample BER-18_52EV. In (a) and (c) the depletion and re-pressurisation steps PPD\#1, PPR\#1 and PPD\#2 are indicated, and specifically highlighted are the axial and radial displacements. The experiment was carried out with a fixed $\Delta S_{\text {rad }} / \Delta P$ ratio of $0 \cdot 4\left(\_47 \mathrm{EV}\right)$ and $1 \cdot 0$ ( $\left.\_52 \mathrm{EV}\right)$. In (a), the radial displacement is positive (compressive), whereas it is negative (expansive) in (c). In (b) and (d) axial and radial strain are plotted against the Terzaghi effective stress specifically for the steps PPD\#1, PPR\#1 and PPD\#2. All changes in strain and stress relevant for the calculation of $C_{\mathrm{m}}$ are provided in the online supplementary material, and plotted in Figs 5 and 6

the considered dependencies are significantly better than $0 \cdot 05$, hence rejecting the null hypothesis, and each correlation coefficient $R^{2}$ is better than 0.9 . The authors' observations hence allow for further interpretation of the linear relations found between $e_{\mathrm{ax}}, e_{\mathrm{rad}}$ and the $\Delta S_{\mathrm{rad}} / \Delta P$ ratio using linear poroelastic theory.

\section{Stress-strain response to depletion of $Z R P-3 A$ samples}

Accepting that a linear relation between all stress-strain components can be found for a model rock such as the Berea sandstone, the authors now turn to the true reservoir sandstone samples obtained from well ZRP-3A, located in the Groningen Field, The Netherlands. In this case, four experiments were performed at room temperature, using synthetic brine as pore fluid, of which two were subjected to triaxial stress, and two to uniaxial-strain boundary conditions. Similar experiments were previously reported by Hol et al. (2018). Here the results are presented in comparison with those for the BER-18 samples.
Plotting $e_{\text {rad }}$ against $\Delta S_{\mathrm{rad}} / \Delta P$ ratio (Fig. 7(a)), and $e_{\mathrm{ax}}$ against $\Delta S_{\text {rad }} / \Delta P$ ratio (Fig. $7(\mathrm{~b})$ ), reveals linear trends for all three depletion cycles $35 \mathrm{MPa}-25 \mathrm{MPa}, 25 \mathrm{MPa}-15 \mathrm{MPa}$, and $15 \mathrm{MPa}-3 \mathrm{MPa}$. Moreover, $e_{\mathrm{rad}}$ is decreasing with increasing $\Delta S_{\mathrm{rad}} / \Delta P$ ratio from positive values at low $\Delta S_{\text {rad }} / \Delta P$ ratio to negative at high $\Delta S_{\text {rad }} / \Delta P$ ratio (Fig. $7($ a)). The measured $e_{\mathrm{ax}}$ is also decreasing with increasing $\Delta S_{\mathrm{rad}} / \Delta P$ ratio, but remains positive. Comparing now the trends obtained for $e_{\mathrm{rad}}$ and $e_{\mathrm{ax}}$ per individual depletion steps, reveals a gradual steepening in slope (also refer to Table 4) resulting from stiffening of the samples by compaction. Indeed, the samples do exhibit inelastic strain as the elastic strain fraction in the range $0 \cdot 57-0 \cdot 86$ (refer to the online supplementary material). It is important to note that the three regression lines intercept the $x$-axis at approximately the same position, hence $\Delta S_{\mathrm{rad}} / \Delta P$ ratio. These observations are qualitatively similar to those made for the BER-18 samples.

The following differences can be observed between the two sample sets. First, the magnitude of $e_{\mathrm{ax}}$ of the ZRP-3A samples is greater than that of the BER-18 per pore pressure 


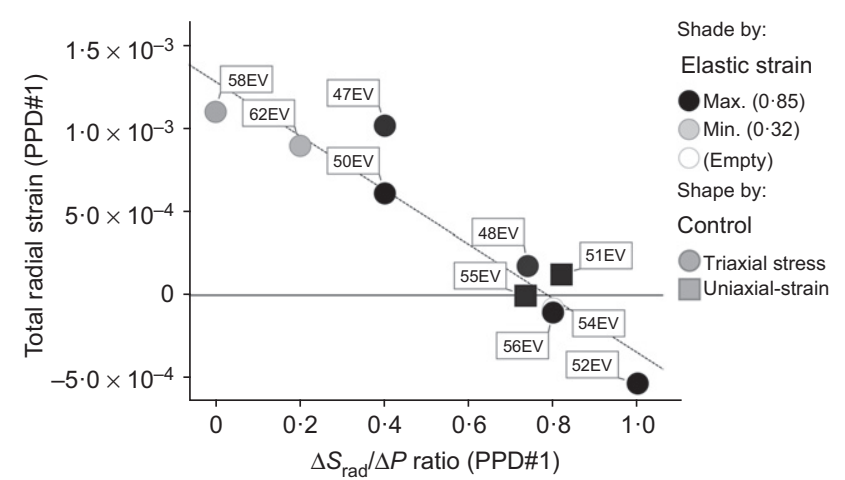

(a)

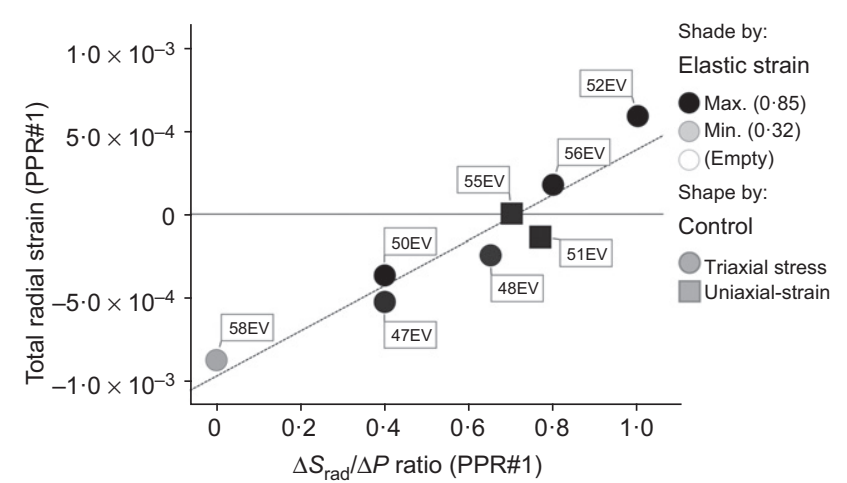

(b)

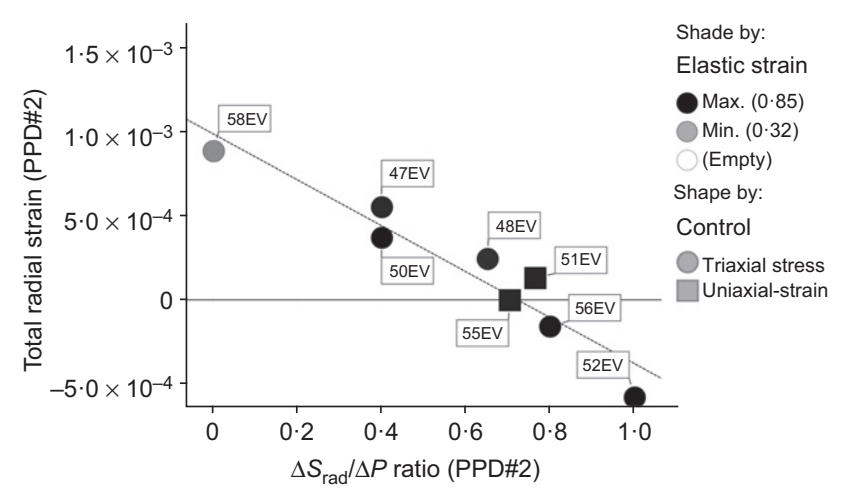

(c)

Fig. 5. Radial strain $e_{\text {rad }}$ against $\Delta S_{\text {rad }} / \Delta P$ ratio for the depletionre-pressurisation-depletion cycles applied to the BER-18 samples. Positive strain is compression. Cycles are identified as PPD\#1, PPR\#1 and PPD\#2, respectively, and strain accordingly. Note the linear dependence in all cases, with an intercept with the $x$-axis between $0 \cdot 6$ and $0 \cdot 8$. The intercept agrees well with the $\Delta S_{\text {rad }} / \Delta P$ ratio for the uniaxial-strain data - that is with the 'measured' $\gamma_{h}$

step. Second, a comparison of the regression statistics (in Table 4) for the ZRP-3A samples with those in Table 4 for the BER-18 samples shows that the quality of the linear fit is less for the ZRP-3A samples. This means the predictive power of any linear model is limited. Third, the recoverability of the axial strain is fundamental for the validity of a truly linear poroelastic model in terms of determining the $C_{1 \text {-uniaxial }}$, or $C_{\mathrm{m}}$ (refer to equations (1) and (2)). The fractional contribution of elastic strain $e_{\mathrm{el}}$ is in the range $0.57-0.86$ with $\Delta S_{\text {rad }} / \Delta P$ ratios in the range $0.5-0.9$ for the ZRP-3A samples, compared to the consistent 0.8 for the BER-18 samples in the same range of $\Delta S_{\mathrm{rad}} / \Delta P$ ratios.

Despite the fact that the errors in the linear fit forced onto the (fewer) ZRP-3A data are larger than those for the BER-18 data, and the observation that more inelastic strain is observed in the case of the ZRP-3A samples, the good linear fit for all $e_{\text {rad }}$ measurements (Fig. 7(a), with $p$-values

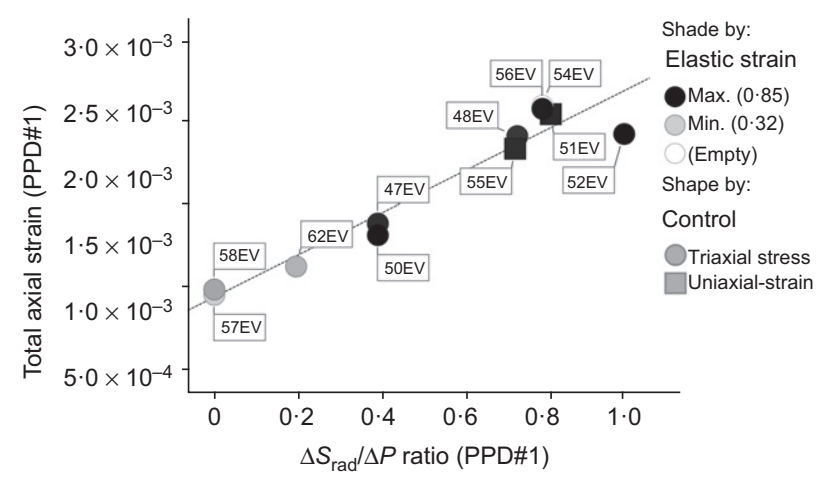

(a)

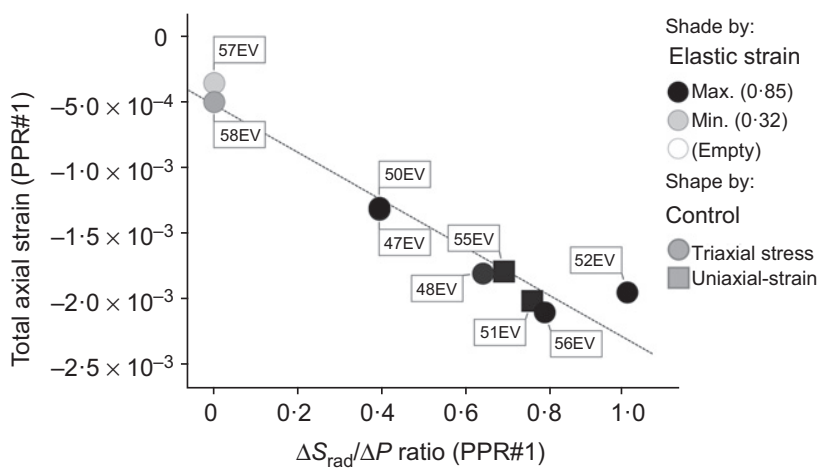

(b)

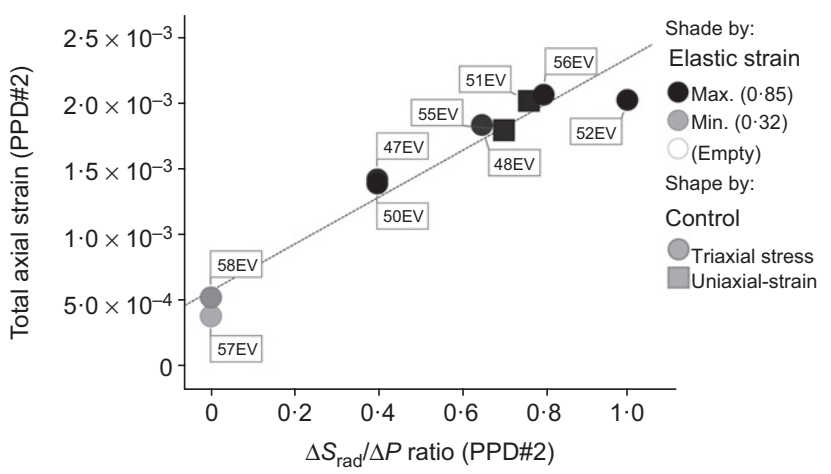

(c)

Fig. 6. Axial strain $e_{\mathrm{ax}}$ against $\Delta S_{\mathrm{rad}} / \Delta P$ ratio for the depletionre-pressurisation-depletion cycles applied to the BER-18 samples. Positive strain is compression. Cycles are identified as PPD\#1, PPR\#1 and PPD\#2, respectively, and strain accordingly. Note the linear dependence in all cases

all significantly lower than $0 \cdot 05$ ) gives confidence in further interpretation of the data in terms of linear poroelastic theory. It is namely the $e_{\text {rad }}$ component that is most critical to assessing the boundary condition of uniaxial-strain.

\section{DISCUSSION}

The experimental work carried out has focused on developing an alternative technique to measure the uniaxialstrain compressibility $C_{\mathrm{m}}$ of cohesive sandstone, with the purpose to aid modelling studies for compaction (drive) and subsidence. The current method determines $C_{\mathrm{m}}$ from triaxial pore pressure depletion tests. Teeuw (1971) developed an approach to correlate hydrostatic and uniaxial-strain conditions, under the assumption that linear poroelasticity would hold for both boundary conditions. However, several problems remained with regards to the application of this idea to the present day. First, most techniques available 


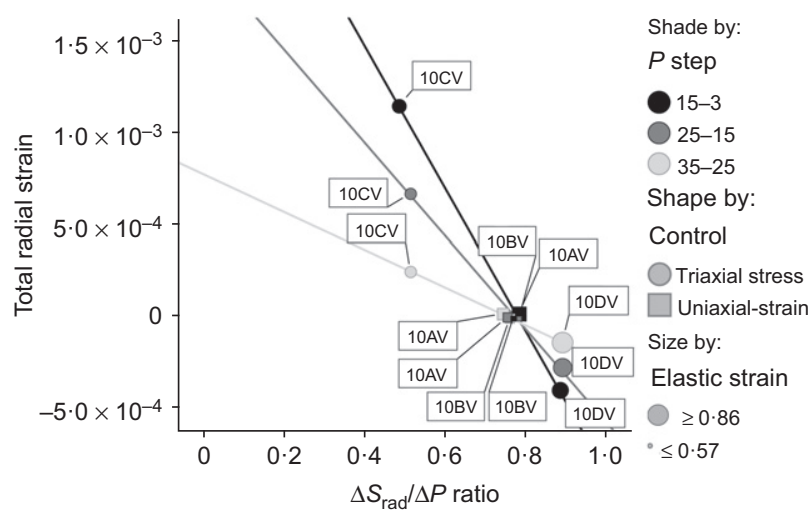

(a)

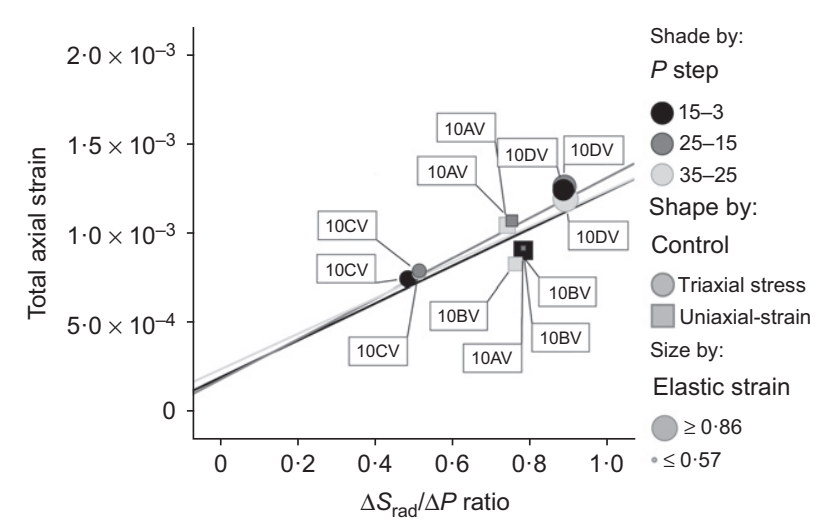

(b)

Fig. 7. (a) Radial strain $e_{\text {rad }}$ against $\Delta S_{\text {rad }} I \Delta P$ ratio for the depletionre-pressurisation-depletion cycles applied to the ZRP-3A samples. Positive strain is compression. Cycles are identified as PPD35-25, PPD25-15 and PPD15-3, respectively, and strain accordingly. Note the linear dependence in all cases, with an intercept with the $x$-axis between 0.6 and 0.8 . The intercept agrees well with the $\Delta S_{\text {rad }} l \Delta P$ ratio for the uniaxial-strain data - that is with the 'measured' $\gamma_{h}$. Note also, the change in slope; (b) Axial strain $e_{\text {ax }}$ against $\Delta S_{\text {rad }} / \Delta P$ ratio for the depletion-re-pressurisation-depletion cycles applied to the ZRP-3A samples. Positive strain is compression. Cycles are identified as PPD35-25, PPD25-15 and PPD15-3, respectively, and strain accordingly. Note the close to linear dependence in all cases

before the mid-1990s were not based on pore pressure depletion testing and hence suffered from an assumption around the magnitude of the effective stress, namely because the Biot-Willis coefficient $\alpha$ is unknown in such a case and the measured strains may be influenced by pre-existing sample damage. Second, the length:diameter ratio of the samples used has long been shorter than $2: 1$, with the consequence that endcap friction affected the sample stress state and hence confining pressure under uniaxial-strain conditions. Finally, and most importantly, the approach of converting hydrostatic compression data to uniaxial-strain conditions suffers from $(a)$ inelastic strain in either one of the boundary conditions, and $(b)$ the need for an independent measurement of the elastic constants. Indeed, experimental studies using both reservoir and outcrop material have shown that boundary conditions can significantly affect the determined compressibility and radial stress response (Teeuw, 1971; Andersen, 1988; Andersen \& Jones, 1985; Rafieepour et al., 2017). Rocks tested under different extreme boundary conditions, for example by crossing the critical state line (Rutter \& Glover, 2012), not subjecting to confinement, or approaching the (hydrostatic) critical pressure (Zhang et al., 1990), can exhibit significant inelastic behaviour. Finally, even rocks such as the Berea sandstone are intrinsically anisotropic (Hart \& Wang, 1995;
Lockner \& Beeler, 2003; Ingraham et al., 2017). Obtaining a complete set of poroelastic parameters therefore requires extensive testing under multiple boundary conditions that may include mechanical loading (Lockner \& Beeler, 2003), acoustic techniques (Wang \& Nur, 1990; Dvorkin \& Nur, 1993) and pressure oscillation methods (Pimienta et al., 2017). The concerns mentioned here have all been addressed by Dudley et al. (2016), which represents the current ISRM-recommended technique. The current authors' approach is fully consistent with Dudley et al. (2016), while it is also in line with the principles set out in the earliest literature on reservoir compressibility (e.g. Hall, 1953; Fatt, 1958; Teeuw, 1971; Andersen \& Jones, 1985; Andersen, 1988). In the following sections, it will be demonstrated how the uniaxial-strain compressibility $C_{\mathrm{m}}$ can indeed be determined under controlled, non-constant triaxial stress boundary conditions, without the need to independently determine the elastic constants $E, v$ and $\alpha$.

\section{Consistency and validity of mechanical data}

The experimental data presented for the BER-18 sandstone samples show that the axial and radial stress-strain responses are related in a linear manner (Figs 4-6), which is consistent with the poroelastic theory adopted in most modelling studies. Moreover, the variation in lateral bounding stress $S_{\text {rad }}$ during depletion seems to control both the radial (Fig. 5) and axial strain (Fig. 6) components. The data also show that uniaxial-strain boundary conditions imposed by active lateral strain control yield radial stress $S_{\text {rad }}$ and axial strain $e_{\mathrm{ax}}$ responses that are in good agreement with the trend obtained from the triaxial pore pressure depletion experiments conducted using BER-18 samples. More specifically, the predicted $\Delta S_{\mathrm{rad}} / \Delta P$ conditions to maintain uniaxial-strain conditions were in agreement to within 0.05 of the $\gamma_{\mathrm{h}}$ determined by direct uniaxial-strain pore pressure depletion tests. For example, the first depletion step would predict an intercept based on the triaxial tests only of 0.77 , compared to the true uniaxial-strain pore pressure depletion test measurements of $0 \cdot 74$ and $0 \cdot 82$. From the overall consistent prediction of the linear, three-dimensional elastic response (refer to statistics in Table 4), it is inferred that the axial strain can indeed be predicted from the interpolated $\gamma_{\mathrm{h}}$ that can be correlated with the linear regression of $e_{\mathrm{ax}}$ plotted against $\Delta S_{\mathrm{rad}} / \Delta P$ ratio. The experimental approach was then applied by taking samples from well ZRP-3A, located in the centre of the subsiding Groningen Field, The Netherlands. In this case, only two triaxial depletion tests were conducted with fixed $\Delta S_{\mathrm{rad}} / \Delta P$ ratios of 0.5 and 0.9 . From the test data, a radial strain intercept of 0.81 was determined by interpolation, compared to 0.83 based on the complete pore pressure depletion step in the case of the true uniaxial-strain compressibility tests. Although the prediction of the $\gamma_{\mathrm{h}}$ by the method based on just two measurements of $e_{\text {rad }}$ can be statistically risky, it appeared solid and consistent for the present purpose. Comparing the experimentally determined values obtained for $\gamma_{\mathrm{h}}$ with values reported by Altmann et al. (2010), shows that the measurements are in good agreement with those for selected field cases. Based on the experiments carried out using the BER-18 and ZRP-3A samples, and the successful prediction of $e_{\mathrm{ax}}$ for uniaxial-strain conditions, the validity of the interpolation-intercept method is confirmed as an alternative for the uniaxial-strain testing, within the reported statistical certainty (Table 4).

\section{Determining the uniaxial-strain compressibility $C_{\mathrm{m}}$}

Following equations (1) and (11) for triaxial stress conditions, and equations (2) and (13) for uniaxial-strain 
conditions, the axial compressibility $C_{1 \text {-uniaxial }}$ can be computed from the elastic constants determined from the test results, and the radial stress response measured during the test. Using now the strain data presented in Figs 5 and 6 for the BER-18 samples, and in Fig. 7 for the ZRP-3A samples, the mean Young's modulus, $E$, and Poisson's ratio, $v$, can be computed by taking the values for $e_{\mathrm{ax}}$ and $e_{\text {rad }}$ for $\Delta S_{\text {rad }} / P=1 \cdot 0$, based on the linear regressing reported in Table 4. The Young's modulus $E$ then follows from $E=\Delta S_{\mathrm{ax}} / \Delta e_{\mathrm{ax}}$, at $\Delta S_{\mathrm{rad}} / P=1 \cdot 0$, and the Poisson's ratio $v=-\Delta e_{\mathrm{rad}} / \Delta e_{\mathrm{ax}}$, at $\Delta S_{\mathrm{rad}} / P=1 \cdot 0$, where $E$ is $8.4 \mathrm{GPa}$ for the total depletion step $35-3 \mathrm{MPa}$ pore pressure, and $v=0 \cdot 18$. Note here that the computed $E$ for depletion is higher than the mean $E$ obtained from the pre-depletion data, which was expected from the consideration of crack closure. Similarly, the BER-18 results yield values for $E$ in the range $11 \cdot 5-13.0 \mathrm{GPa}$, and $v=0 \cdot 13-0 \cdot 17$, which is again higher than the 7-10 GPa reported here, and now more consistent with the range 10-20 GPa reported in the literature (Hart \& Wang, 1995; Delle Piane \& Sarout, 2016). Taking the computed values for $E$ and $v$, combined with the interpolated $\gamma_{\mathrm{h}}$ values for the corresponding pore pressure depletion step and sample series, the axial compressibility $C_{1-\text { uniaxial }}$ is now calculated, or the equivalent uniaxial-strain compressibility $C_{\mathrm{m}}$ under boundary conditions of uniaxial-strain, by inserting the obtained values into equation (2). For the BER-18 samples, the authors compute, for the three cycles PPD\#1, PPR\#1 and PPD\#2, respective $C_{\mathrm{m}}$ values of $8.20 \times 10^{-5} \mathrm{MPa}^{-1}$, $6.92 \times 10^{-5} \mathrm{MPa}^{-1}$ and $7.02 \times 10^{-5} \mathrm{MPa}^{-1}$. Comparing these with the $C_{\mathrm{m}}$ values determined directly, respectively $7 \cdot 83 \times 10^{-5}$ for PPD\#1, $6.33 \times 10^{-5}$ for PPR\#1 and $6 \cdot 33 \times 10^{-5}$ for PPD\#2, shows that the $C_{\mathrm{m}}$ values attained through the application of the interpolation-intercept method are in good agreement with those from direct measurements. For the total depletion of the ZRP-3A samples, a mean $C_{\mathrm{m}}$ of $1.12 \times 10^{-4} \mathrm{MPa}^{-1}$ is computed, versus the direct determination by uniaxial-strain pore pressure depletion of $9.66 \times 10^{-5} \mathrm{MPa}^{-1}$.

Based on the quality of the regression - that is $R^{2}$ and the $p$-value, it is now also possible to extend the treatment and provide the prediction of $C_{\mathrm{m}}$ with a measure of confidence. This presently goes beyond the scope of demonstrating the interpolation-intercept method. Note that by taking the true uniaxial-strain data into account in the linear regression fit, implicit deviations beyond obviously large differences are captured by the regression statistics.

\section{Specific requirements and constraints of the interpolation-intercept method}

Although the authors have shown that their technique can be applied to both cohesive outcrop material and reservoir material from a field that is actually subsiding, the success of the interpolation-intercept method in determining $C_{\mathrm{m}}$ is dependent on conforming to several key requirements. First, the method is based on triaxial testing but solely by pore pressure depletion. Hence, triaxial compressive strength measurements are not suitable for the determination of $C_{\mathrm{m}}$. Second, $e_{\text {rad }}$ can only be obtained reliably by interpolation, and never by extrapolation. Therefore, the $\Delta S_{\text {rad }} / \Delta P$ ratio values adopted for the control of the triaxial pore pressure depletion tests should be chosen such that both positive and negative $e_{\mathrm{rad}}$ can be measured, whereby values of $\Delta S_{\mathrm{rad}} / \Delta P$ between 0.4 and 0.9 are most realistic based on linear poroelastic considerations. Recall that the tests conducted with a value of $0 \cdot 0$ resulted in lower fractional contributions of axial elastic strain (refer to Fig. 5), which demonstrates that such tests are indeed less suitable. This again highlights one of the concerns with utilising hydrostatic compressibility data (equivalent to $\Delta S_{\mathrm{rad}} / \Delta P=1$ ) to the uniaxial-strain compressibility. Third, properly calibrated test systems should be used following the specifications set out by the ISRM suggested method (Dudley et al., 2016). Interestingly, it is noted here that the interpolation-intercept method is, in fact, ideal to assess the technical quality of such systems, because it allows for an independent check of uniaxial-strain test results. To do this, both triaxial stress and uniaxial-strain pressure depletion tests should be conducted on similar rock samples, in the manner presented in this paper. This has been demonstrated here for the ZRP-3A samples. Fourth, the material should be sufficiently elastic to guarantee that the three-dimensional poroelastic behaviour can be characterised in a manner consistent with the theory formulated in equations (1) and (2). In practice, this means that the samples should be sufficiently cohesive and elastic. As shown by the experiments using the BER-18 samples, but certainly with the ZRP-3A samples, inelastic strain indeed has an impact on the strains and stress measured during depletion, but not on the intercept with the $\Delta S_{\mathrm{rad}} / \Delta P$ axis when the fractional contribution of elastic strain $e_{\mathrm{el}}$ is at least $0 \cdot 5$. Despite the good agreement between theory and measurements presented here, the authors emphasise that their study has not identified the exact cohesion limit below which the three-dimensional poroelastic coupling starts to break down because of inelastic strain accumulation. This could be the subject of future study.

\section{CONCLUSIONS}

The aim of this study was to develop an alternative test method to determine the uniaxial-strain compressibility $C_{\mathrm{m}}$. This method is complementary to the methods oedometric or pore pressure depletion testing under active radial strain control, or radial confinement. This parameter is crucial for the assessment of reservoir compaction, compaction drive effects and potentially subsidence. The authors have reported pore pressure depletion tests conducted under triaxial stress control and uniaxial-strain control, to compare with a theoretical poroelastic framework, and the findings are summarised as follows.

(a) Triaxial pore pressure depletion experiments carried out here yield radial stress $S_{\mathrm{rad}}$ and axial strain $e_{\mathrm{ax}}$ responses that are in good agreement with data from tests using actively controlled uniaxial-strain boundary conditions. The predicted $\Delta S_{\mathrm{rad}} / \Delta P$ conditions to maintain uniaxial-strain conditions were to within 0.05 of the $\gamma_{\mathrm{h}}$ determined by direct uniaxial-strain pore pressure depletion tests. For the BER-18 samples, the interpolated point of zero radial strain for the first depletion step was $\Delta S_{\text {rad }} / \Delta P$ ratio of $0 \cdot 77$, compared to depletion path constants $\gamma_{\mathrm{h}}$ measured from true uniaxial-strain pore pressure depletion tests of 0.74 and 0.82 . The re-pressurisation, and second depletion step, following yielded similar results.

(b) Using the laboratory data, in combination with theory developed here for poroelastic response of reservoir rocks under both triaxial stress and uniaxial-strain boundary conditions allowed for the computation of the axial compressibility $C_{1}$, specifically $C_{1-\text { triaxial }}$ or $C_{1 \text {-uniaxial }}$. Average values obtained for BER-18 samples were $8.20 \times 10^{-5} \mathrm{MPa}^{-1}(\mathrm{PPD \# 1}), 6.92 \times 10^{-5} \mathrm{MPa}^{-1}$ (PPR\#1) and 7.02 $\times 10^{-5} \mathrm{MPa}^{-1}$ (PPD\#2) from triaxial, compared to a respective $7 \cdot 83 \times 10^{-5}$, $6 \cdot 33 \times 10^{-5}$ and $6 \cdot 33 \times 10^{-5}$, obtained directly by 
uniaxial strain testing. ZRP-3A samples yield $1 \cdot 12 \times 10^{-4} \mathrm{MPa}^{-1}$, as against $9.66 \times 10^{-5} \mathrm{MPa}^{-1}$ determined directly by the uniaxial-strain protocol. About $80 \%$ of the strain response measured around the intercept point was recoverable.

(c) The interpolation-intercept method proposed here has proved successful in its test against real reservoir rocks undergoing compaction in the field. The horizontal depletion path constant $\gamma_{\mathrm{h}}$, and the uniaxial-strain compressibility $C_{\mathrm{m}}$ obtained by using this method agree well with those measured directly using a direct uniaxial-strain compressibility test. The authors therefore conclude that $C_{\mathrm{m}}$ can be reliably approximated for poroelastic rocks, at stress conditions below the critical state line. The chosen interpolation-intercept method can be used in practice when no uniaxial-strain capabilities are available, or when control tests are required to test for the technical performance and quality of the radial strain control system, as part of the laboratory set-up.

(d) Specific requirements set out in the ISRM suggested method for uniaxial-strain compressibility testing, as well as by linear poroelastic theory do apply to the interpolation-intercept method, and should be considered when implementing it.

The Mohr-Coulomb cohesion limit, below which a material could be considered sufficiently cohesive, is not known at present, and could be the subject of future research.

\section{APPENDIX. THEORY OF COMPRESSIBILITY}

Taking into consideration the recent contribution by Zimmerman (2017), here a number of practical equations are derived to compare rock compressibility under triaxial and uniaxial-strain lateral boundary conditions, with the purpose to enable the determination of the uniaxial-strain compressibility out of pore pressure depletion tests conducted under triaxial stress conditions. Following Wang (2000), strain components $e_{1}, e_{2}$ and $e_{3}$ for an isotropic linear poroelastic rock can be written for the principal coordinates that also represent the principal loading directions in a triaxial vessel

$$
\begin{aligned}
& e_{1}=\frac{\sigma_{1}}{E}-\frac{v}{E} \sigma_{2}-\frac{v}{E} \sigma_{3}+\frac{\alpha P}{3 K} \\
& e_{2}=-\frac{v}{E} \sigma_{1}+\frac{\sigma_{2}}{E}-\frac{v}{E} \sigma_{3}+\frac{\alpha P}{3 K} \\
& e_{3}=-\frac{v}{E} \sigma_{1}-\frac{v}{E} \sigma_{2}+\frac{\sigma_{3}}{E}+\frac{\alpha P}{3 K}
\end{aligned}
$$

here, $\sigma_{1}, \sigma_{2}$ and $\sigma_{3}$, are three principal stresses; $E$ is the Young's modulus; $v$ is the Poisson's ratio; $\alpha$ is the Biot-Willis coefficient; and $K$ is the bulk modulus. In the case of a conventional triaxial vessel system, the confining pressure equals both $\sigma_{2}$ and $\sigma_{3}$, and hence $\sigma_{1} \geq \sigma_{2}=\sigma_{3}$, and $e_{1} \geq e_{2}=e_{3}$. Equations (3)-(5) are now reduced to expressions for $e_{1}$ and $e_{3}$ only, to represent the measured strains in the vessel, thereby also taking $K=E /[3(1-2 v)]$, and rewriting to

$$
\begin{aligned}
& e_{1}=\frac{\sigma_{1}}{E}-\frac{2 v}{E} \sigma_{3}+\alpha P \frac{1-2 v}{E} \\
& e_{3}=\frac{\sigma_{3}}{E}-\frac{v}{E}\left(\sigma_{1}+\sigma_{3}\right)+\alpha P \frac{1-2 v}{E}
\end{aligned}
$$

For triaxial stress conditions, pore pressure depletion under constant $\sigma_{1}$ is now considered, hence $\sigma_{1,1}=\sigma_{1, \text { ref }}$, and changing $\sigma_{3}$, hence $\sigma_{3,1} \neq \sigma_{3, \text { ref. }}$ It follows that

$$
\Delta e_{1}=-\frac{2 v}{E}\left(\sigma_{3,1}-\sigma_{3, \text { ref }}\right)+\frac{\alpha(1-2 v)}{E}\left(P_{1}-P_{\text {ref }}\right)
$$

$$
\Delta e_{3}=\frac{1-v}{E}\left(\sigma_{3,1}-\sigma_{3, \text { ref }}\right)+\frac{\alpha(1-2 v)}{E}\left(P_{1}-P_{\text {ref }}\right)
$$

Realising and accepting that most cohesive reservoir sandstones have a layered structure, modelling studies often assume isotropic linear poroelasticity. Under such an assumption, a change in pore pressure at constant external stress results in a change in $e_{1}$ that equals the change in $e_{3}$ - that is the rock deforms only by dilation. Taking $\Delta e_{1}=e_{1,1}-e_{1, \text { ref }}$ and $\Delta e_{3}=e_{3,1}-e_{3, \text { ref }}$, with $\sigma_{1,1}=\sigma_{1, \text { ref, }}$, and $\sigma_{3,1}=\sigma_{3, \text { ref }}$, it namely turns out that

$$
\Delta e_{1}=\Delta e_{3}=\frac{\alpha(1-2 v)}{E} \Delta P
$$

In this paper, however, the authors specifically focus on the role of radial stress in the assessment of the axial compressibility, notably to deduce from triaxial measurements the uniaxial-strain compressibility $C_{\mathrm{m}}$. To explore the lateral boundary conditions of triaxial stress and uniaxial-strain employed in these tests, first the derivative is taken of $\Delta e_{1}$ and $\Delta e_{3}$ to $\Delta P$

$$
\begin{gathered}
\Delta e_{3}=\frac{(1-v)}{E}\left(\sigma_{3,1}-\sigma_{3, \text { ref }}\right)+\frac{\alpha(1-2 v)}{E}\left(P_{1}-P_{\text {ref }}\right) \rightarrow \\
C_{3-\text { triaxial }}=\frac{\Delta e_{3}}{\Delta P}=\frac{(1-v)}{E} \frac{\Delta \sigma_{3}}{\Delta P}+\frac{\alpha(1-2 v)}{E}
\end{gathered}
$$

and

$$
\begin{gathered}
\Delta e_{1}=-\frac{2 v}{E}\left(\sigma_{3,1}-\sigma_{3, \text { ref }}\right)+\frac{\alpha(1-2 v)}{E}\left(P_{1}-P_{\text {ref }}\right) \rightarrow \\
C_{1-\text { triaxial }}=\frac{\Delta e_{1}}{\Delta P}=-\frac{2 v}{E} \frac{\Delta \sigma_{3}}{\Delta P}+\frac{\alpha(1-2 v)}{E}
\end{gathered}
$$

For the special case of uniaxial-strain, $\Delta e_{3}=0$, the balance of forces dictates

$$
\begin{aligned}
\Delta e_{3}=0 & =\frac{(1-v)}{E}\left(\sigma_{3,1}-\sigma_{3, \text { ref }}\right)+\frac{\alpha(1-2 v)}{E}\left(P_{1}-P_{\text {ref }}\right) \rightarrow \\
\frac{\Delta \sigma_{3}}{\Delta P} & =-\frac{\alpha(1-2 v)}{(1-v)}=\gamma_{\mathrm{h}}
\end{aligned}
$$

The property $\gamma_{\mathrm{h}}$ is known in the literature as the horizontal depletion path constant that expresses the change in radial stress in response to an imposed change in pore pressure under uniaxialstrain boundary conditions. Similarly, equation (13) can be inserted into equation (1), which yields the axial compressibility $C_{1 \text {-uniaxial }}$ under uniaxial-strain boundary conditions, here assumed to be the uniaxial-strain compressibility $C_{\mathrm{m}}$ (Geertsma, 1973a)

$$
\begin{gathered}
\Delta e_{1}=-\frac{2 v}{E} \Delta \sigma_{3}+\frac{\alpha(1-2 v)}{E} \Delta P \rightarrow \\
C_{1-\text { uniaxial }}=C_{\mathrm{m}}=\frac{\Delta e_{1}}{\Delta P}=-\frac{2 v \gamma_{\mathrm{h}}}{E}+\frac{\alpha(1-2 v)}{E}
\end{gathered}
$$

Equation (14) has the simple shape $C_{1 \text {-uniaxial }}=A \gamma_{\mathrm{h}}+B$ (see also Hettema et al. (2000)), where the exact values of the elastic constants are assumed to be constant over the range of applied stress, pressure and temperature conditions. This approach is considered valid for depletion of many reservoirs consisting of cohesive sandstone. Taking now for representative cohesive reservoir sandstone, $\alpha=0.8-1 \cdot 0, \gamma_{\mathrm{h}}=0 \cdot 7, v=0 \cdot 15-0 \cdot 25$ and $E=10-20 \mathrm{GPa}$, the compressibility $C_{1 \text {-uniaxial }}$ is in the range from $1.5 \times 10^{-5} \mathrm{MPa}^{-1}$ to $1.75 \times 10^{-5} \mathrm{MPa}^{-1}$, which is roughly consistent with data reported in the literature (Rhett \& Teufel, 1992; Hettema et al., 2000; Hol et al., 2015; Roholl et al., 2016). Given the relationship between the radial stress change, $\Delta \sigma_{3}$, and the radial strain, $\Delta e_{3}$, as expressed in equations (11) and (13), it is possible to approach zero $\Delta e_{3}$ by varying $\Delta \sigma_{3}$ systematically.

The applied experimental conditions are here referred to as the axial stress $S_{\text {ax }}$, which equals $\sigma_{1}$, the radial stress $S_{\text {rad }}$, which equals $\sigma_{2}$ and $\sigma_{3}$, and pore pressure $P$. Using the linear nature of the constitutive response, only two measurements are required to determine the intercept of $\Delta S_{\mathrm{rad}} / \Delta P$ at $\Delta e_{3}=\Delta e_{\mathrm{rad}}=0$, which is in fact the $\gamma_{\mathrm{h}}$ for the case of equation (13) that can then be inserted into 
equation (14) to compute $C_{1 \text {-uniaxial, }}$, or $C_{\mathrm{m}}$ from a modelling perspective. Using this experimental strategy is only possible for rocks that are close to isotropic linear elastic, and it can be applied without quantification of the elastic constants.

\section{NOTATION \\ $C_{\mathrm{m}} \quad$ uniaxial-strain compressibility $\left(\mathrm{MPa}^{-1}\right)$ \\ $C_{1 \text {-triaxial }}, C_{3 \text {-triaxial }} \quad$ axial and radial compressibilities under triaxial stress boundary conditions $\left(\mathrm{MPa}^{-1}\right)$ \\ $C_{1-\text { uniaxial }}$ axial compressibility under uniaxial strain boundary conditions $\left(\mathrm{MPa}^{-1}\right)$ \\ $E$ Young's modulus $(\mathrm{GPa})$ \\ $e_{1}, e_{2}, e_{3}$ principal strain components \\ $e_{\mathrm{ax}}, e_{\mathrm{rad}}$ axial and radial strains from experimental data \\ $K$ bulk modulus (GPa) \\ $P$ pore pressure $(\mathrm{MPa})$ \\ $P_{\mathrm{c}} \quad$ confining pressure $(\mathrm{MPa})$ \\ $P_{\text {dep }} \quad$ final experimental depletion pressure (MPa) \\ $P_{\text {ini }} \quad$ initial (pre-depletion) experimental pore pressure (MPa) \\ $S_{\mathrm{ax}}, S_{\mathrm{rad}}$ applied (experimental) axial and radial stresses on the sample (MPa) \\ $V_{\mathrm{b}}$ bulk volume $\left(\mathrm{m}^{3}\right)$ \\ $V_{\mathrm{p}}$ pore volume $\left(\mathrm{m}^{3}\right)$ \\ $\alpha$ Biot-Willis coefficient \\ $\gamma_{\mathrm{h}}$ horizontal depletion path constant under uniaxial-strain boundary conditions \\ $\Delta e_{1}, \Delta e_{3} \quad$ change in principal strains $e_{1}$ and $e_{3}$ relative to references $e_{1, \text { ref }}$ and $e_{3, \text { ref }}$ \\ $\Delta S_{\mathrm{rad}} / \Delta P$ ratio, imposed change in $S_{\mathrm{rad}}$ per change in $\Delta P$, equals $\gamma_{\mathrm{h}}$ under uniaxial-strain conditions $v$ Poisson's ratio \\ $\sigma_{1}, \sigma_{2}, \sigma_{3}$ principal stresses (MPa)}

\section{REFERENCES}

Altmann, J. B., Müller, T. M., Müller, B. I. R., Tingay, M. R. P. \& Heidbach, O. (2010). Poroelastic contribution to the reservoir stress path. Int. J. Rock Mech. Min. Sci. 47, No. 7, 1104-1113.

Andersen, M. A. (1988). Predicting reservoir-condition PV compressibility from hydrostatics stress laboratory data. SPE Reservoir Engng 3, No. 03, 1078-1082.

Andersen, M. A. \& Jones, F. O., Jr. (1985). A comparison of hydrostatic-stress and uniaxial-strain pore-volume compressibilities using nonlinear elastic theory. In Research and engineering applications in rock masses: proceedings of the 26th US symposium on rock mechanics (ed. E. Ashworth), vol. 1, pp. 403-412. Rotterdam, the Netherlands: Balkema.

Biot, M. A. (1941). General theory of three-dimensional consolidation. J. Appl. Phys. 12, No. 2, 155-164.

Bouteca, M. (1992). Elements of poro-elasticity for reservoir engineering. Revue de l'Institut Français du Pétrole 47, No. 4, 479-490.

Burbey, T. J. (1999). Effects of horizontal strain in estimating specific storage and compaction in confined and leaky aquifer systems. Hydrogeol. J. 7, No. 6, 521-532.

Delle Piane, C. \& Sarout, J. (2016). Effects of water and supercritical $\mathrm{CO}_{2}$ on the mechanical and elastic properties of Berea sandstone. Int. J. Greenh. Gas Con. 55, 209-220.

Donnelly, L. J. (2009). A review of international cases of fault reactivation during mining subsidence and fluid abstraction. Q. J. Engng Geol. Hydrogeol. 42, No. 1, 73-94.

Dudley, J. W., Brignoli, M., Crawford, B. R., Ewy, R. T., Love, D. K., Mclennan, J. D., Ramos, G. G., Shafer, J. L., Sharf-Aldin, M. H., Siebrits, E., Boyer, J. \& Chertov, M. A. (2016). ISRM suggested method for uniaxial-strain compressibility testing for reservoir geomechanics. Rock Mech. Rock Engng 49, No. 10, 4153-4178.

Dvorkin, J. \& Nur, A. (1993). Dynamic poroelasticity: a unified model with the squirt and the Biot mechanisms. Geophysics 58. No. 4, 524-533.

Eshelby, J. D. (1957). The determination of the elastic field of an ellipsoidal inclusion, and related problems. Proc. R. Soc. A 241, No. 1226, 376-396.
Fatt, I. (1958). Pore volume compressibilities of sandstone reservoir rocks. J. Petrol. Technol. 10, No. 3, 64-66.

Geertsma, J. (1973a). A basic theory of subsidence due to reservoir compaction: the homogeneous case. Verhandelingen Koninklijk Nederlands Geolologisch en Mijnbouwkundig Genootschap 28, 43-62.

Geertsma, J. (1973b). Land subsidence above compacting oil and gas reservoirs. J. Petrol. Technol. 25, 734-744.

Gill, J. C. \& Malamud, B. D. (2014). Reviewing and visualizing the interactions of natural hazards. Rev. Geophys. 52, No. 4, 680-722.

Hall, H. N. (1953). Compressibility of reservoir rocks. Petrol. Trans. 168, 309-311.

Hart, D. J. \& Wang, H. F. (1995). Laboratory measurements of a complete set of poroelastic moduli for Berea sandstone and Indiana limestone. J. Geophys. Res. 100, No. B9, 17741-17751.

Hettema, M. H. H., Schutjens, P. M. T. M., Verboom, B. J. M. \& Gussinklo, H. J. (2000). Production-induced compaction of a sandstone reservoir: the strong influence of stress path. SPE Reserv. Eval. Engng 3, No. 4, 342-347.

Hol, S., Mossop, A. P., Van Der Linden, A. J., Zuiderwijk, P. M. M. \& Makurat, A. H. (2015). Long-term compaction behavior of Permian sandstones - an investigation into the mechanisms of subsidence in the Dutch Wadden sea. Proceedings of the 49th US rock mechanics/geomechanics symposium, San Francisco, CA, USA, paper ARMA-2015-618.

Hol, S., Van Der Linden, A., Bierman, S., Marcelis, F. \& Makurat, A. (2018). Rock physical controls on productioninduced compaction in the Groningen Field. Sci. Rep. 8, No. 1, 7156.

Ingraham, M. D., Bauer, S. J., Issen, K. A. \& Dewers, T. A. (2017). Evolution of permeability and Biot coefficient at high mean stresses in high porosity sandstone. Int. J. Rock Mech. Min. Sci. 96, 1-10.

Jacob, C. E. (1940). On the flow of water in an elastic artesian aquifer. Eos, Trans. Am. Geophys. Un. 21, No. 2, 574-586.

Lockner, D. A. \& Beeler, N. M. (2003). Stress-induced anisotropic poroelasticity response in sandstone. 16th ASCE engineering mechanics conference, Seattle, WA, USA.

Pimienta, L., Fortin, J. \& Guéguen, Y. (2017). New method for measuring compressibility and poroelasticity coefficients in porous and permeable rocks. J. Geophys. Res.: Solid Earth 122, No. 4, 2670-2689.

Rafieepour, S., Miska, S. Z., Ozbayoglu, E., Yu, M., Takach, N. E., Zhang, J. \& Majidi, R. (2017). Experimental study of reservoir stress path and hysteresis during depletion and injection under different deformational conditions. 51st US rock mechanics/ geomechanics symposium, San Francisco, CA, USA.

Rhett, D. W. \& Teufel, L. W. (1992). Effect of reservoir stress path on compressibility and permeability of sandstones. SPE annual technical conference and exhibition, Washington, DC, USA.

Roholl, J. A., Van Thienen-Visser, K. \& Breunese, J. N. (2016). Translating laboratory compaction test results to field scale. In 50th US rock mechanics / geomechanics symposium 2016, vol. 4, pp. 3180-3186. Red Hook, NY, USA: Curran Associates.

Rutter, E. H. \& Glover, C. T. (2012). The deformation of porous sandstones; are Byerlee friction and the critical state line equivalent? J. Struct. Geol. 44, Supplement C, $129-140$.

Schutjens, P. M. T. M., De Ruig, H., Van Muster, J. G., Sayers, C. M. \& Whitworth, J. L. (1996). Production-induced compaction of the Brent field: an experimental approach. SPE Formation Eval. 11, No. 2, 99-107.

Shahri, M. P. \& Miska, S. Z. (2014). Experimental investigation of reservoir stress path hysteresis during production-injection periods. In $A S M E 201433 r d$ international conference on ocean, offshore and Arctic engineering. Volume 5: materials technology; petroleum technology, paper OMAE2014-24653, V005T11A031. New York, NY, USA: American Society of Mechanical Engineers.

Teeuw, D. (1971). Prediction of formation compaction from laboratory compressibility data. Soc. Petrol. Engrs J. 11, No. 03, 263-271.

Teufel, L. W., Rhett, D. W. \& Farrell, H. E. (1991). Effect of reservoir depletion and pore pressure drawdown on in situ stress and deformation in the Ekofisk field, North sea. In Rock mechanics 
as a multidisciplinary science: proceedings of the 32nd US symposium (ed. J.-C. Roegiers), pp. 63-72. Boca Raton, FL, USA: CRC Press.

Wang, H. F. (2000). Theory of linear poroelasticity with applications to geomechanics and hydrogeology. Princeton, NJ, USA: Princeton University Press.

Wang, Z. \& Nur, A. (1990). Wave velocities in hydrocarbon-saturated rocks: experimental results. Geophysics 55, No. 6, 723-733.
Zhang, J., Wong, T. F. \& Davis, D. M. (1990). Micromechanics of pressure-induced grain crushing in porous rocks. J. Geophys. Res.: Solid Earth 95, No. B1, 341-352.

Zimmerman, R. W. (1991). Compressibility of sandstones. Amsterdam, the Netherlands: Elsevier.

Zimmerman, R. W. (2017). Pore volume and porosity changes under uniaxial strain conditions. Transp. Porous Media 119, No. 2, 481-498. 